\title{
MicroRNA-375 inhibits tumour growth and metastasis in oesophageal squamous cell carcinoma through repressing insulin-like growth factor 1 receptor
}

\author{
Kar Lok Kong, ${ }^{1,2}$ Dora Lai Wan Kwong, ${ }^{1,2}$ Tim Hon-Man Chan, ${ }^{1,2}$ Simon Ying-Kit Law, ${ }^{3}$ \\ Leilei Chen, ${ }^{1,2}$ Yan Li, ${ }^{1,2}$ Yan-Ru Qin, ${ }^{4}$ Xin-Yuan Guan ${ }^{1,2,5}$
}

\begin{abstract}
- Additional materials are published online only. To view these files please visit the journal online (http://gut.bmj. com)

${ }^{1}$ Department of Clinical Oncology, The University of Hong Kong, Hong Kong, China ${ }^{2}$ Center for Cancer Research, The University of Hong Kong, Hong Kong, China

${ }^{3}$ Department of Surgery, The University of Hong Kong, Hong Kong, China

${ }^{4}$ Department of Clinical Oncology, the First Affiliated Hospital, Zhengzhou University, Zhengzhou, China

${ }^{5}$ State Key Laboratory of Oncology in Southern China, Cancer Center, The First Affiliated Hospital, Sun Yat-Sen University, Guangzhou, China
\end{abstract}

\section{Correspondence to}

Xin-Yuan Guan, Department of Clinical Oncology, the University of Hong Kong, Room L10-56, Laboratory Block, 21 Sassoon Road, Pokfulam, Hong Kong, China; xyguan@hkucc.hku.hk

Revised 10 May 2011 Accepted 2 June 2011 Published Online First 3 August 2011

\section{ABSTRACT}

Background To understand the involvement of microRNA (miRNA) in the development and progression of oesophageal squamous cell carcinoma (ESCC), miRNA profiles were compared between tumour and corresponding non-tumour tissues.

Methods miRCURY LNA array was used to generate miRNA expressing profile. Real-time quantitative PCR was applied to detectthe expression of miR-375 in ESCC samples and its correlation with insulin-like growth factor 1 receptor (IGF1R). Methylation-specific PCR was used to study the methylation status in the promoter region of miR-375. The tumour-suppressive effect of miR-375 was determined by both in-vitro and in-vivo assays.

Results The downregulation of miR-375 was frequently detected in primary ESCC, which was significantly correlated with advanced stage $(p=0.003)$, distant metastasis $(p<0.0001)$, poor overall survival $(p=0.048)$ and disease-free survival $(p=0.0006)$. Promoter methylation of miR-375 was detected in 26 of 45 (57.8\%) ESCC specimens. Functional assays demonstrated that miR-375 could inhibit clonogenicity, cell motility, cell proliferation, tumour formation and metastasis in mice. Further study showed that miR-375 could interact with the 3 '-untranslated region of IGF1R and downregulate its expression. In clinical specimens, the expression of IGF1R was also negatively correlated with miR-375 expression ( $p=0.008$ )

Conclusions This study demonstrates that miR-375 has a strong tumour-suppressive effect through inhibiting the expression of IGF1R. The downregulation of miR-375, which is mainly caused by promoter methylation, is one of the molecular mechanisms involved in the development and progression of ESCC.

Oesophageal cancer is the sixth most common cause of death and eighth most common cancer in the world. ${ }^{1}$ Oesophageal cancer can be divided into two main forms: oesophageal squamous cell carcinoma (ESCC) and adenocarcinoma. Adenocarcinoma is common in western countries while ESCC is predominant in east Asia, especially in China. ${ }^{2}$ The pathogenesis of oesophageal cancer still remains unclear although chronic irritation, inflammation and increased cell turnover, which may be caused by gastroesophageal reflux or smoking, have been associated with the carcinogenic process. ${ }^{1}$ In addition, genetic predisposition is another important aetiological factor. Risk et $a l^{3}$ showed that genomic instability at chromosome

\section{Significance of this study}

What is already known about this subject?

- miR-375 is shown to be involved in glucose regulation of insulin gene expression and $\beta$-cell growth.

- Recent profile studies showed that miR-375 is downregulated in gastric cancer and hepatocellular carcinoma implying the microRNA may play an important role in cancer.

- The downregulation of miR-375 may be related to the expression of $\beta$-catenin genes in hepatocellular carcinoma.

What are the new findings?

- miR-375 is strongly downregulated in ESCC, which associated with advanced stage, distant metastasis, poor overall and disease-free survival of ESCC patients.

- By methylation-specific PCR and BGS, we demonstrated that the downregulation of miR375 is caused by the hypermethylation of the promoter region.

- Ectopic expression of miR-375 is sufficient to inhibit tumour cell growth and metastasis in vitro and in vivo. miR-375 may be an important tumour-suppressive miRNA in ESCC.

- miR-375 directly targets IGF1R indicating that it may have significant impact on the Akt pathway. In addition, we showed that the expression of miR-375 is negatively correlated with the expression of IGF1R in eight ESCC cell lines and 60 pairs of ESCC samples.

How might it impact on clinical practice in the foreseeable future?

- This study demonstrated that miR-375 plays a significant role in ESCC tumour cell growth and metastasis by inhibiting an important gene, IGF1R, indicating that miR-375 may be a potential therapeutic target for ESCC patients. In addition, the downregulation of miR-375 correlated with poor overall and disease-free survival suggesting that miR-375 can be a novel biomarker to improve the management of ESCC patients.

17q25 predisposes patients to ESCC. Despite advances in the diagnosis and treatment of this cancer, the 5-year overall survival rate is still less than 
14\%. ${ }^{4}$ Therefore, there is a great need to discover more biomarkers and therapeutic targets for oesophageal cancer patients.

Micro-RNA (miRNA) are a class of single-stranded, non-coding RNA only 17-25 ribonucleotides long. ${ }^{5}$ They are transcribed by RNA polymerase II in hairpin structures and are processed by RNAse III Drosha into long pre-miRNA. The precursors are then processed by RNAse III Dicer to be mature miRNA, which are able to bind to the $3^{\prime}$-untranslated region (UTR) of target messenger RNA through completelt or partially complementarily causing degradation or translation repression, respectively. ${ }^{6}$ By downregulating the target mRNA, miRNA play important roles in the regulation of important processes such as proliferation, differentiation, apoptosis and development. ${ }^{7}$ Several miRNA have been reported in the development of ESCC. miR-21 has been reported to be frequently upregulated in ESCC cell lines and tissues, which regulates cell proliferation and invasion through suppressing PTEN and PDCD4. ${ }^{8}$ On the other hand, miR-203 was reported to be downregulated in ESCC. ${ }^{9}$ Nevertheless, further knowledge about the molecular mechanisms of ESCC miRNA is needed to provide deeper insights for better therapeutic opportunities for ESCC patients. In the present study, a microarray strategy was applied to identify differentially expressed miRNA in ESCC cells by comparing miRNA profiles between tumour and paired non-tumour tissues. Twenty-two differentially expressed miRNA were detected. The tumour-suppressing function and mechanism of miR-375, which was frequently downregulated in ESCC, was further characterised.

\section{MATERIALS AND METHODS \\ ESCC cell lines and clinical samples}

Six Japanese ESCC cell lines (KYSE30, KYSE140, KYSE180, KYSE410, KYSE510 and KYSE520) were obtained from DSMZ, the German Resource Centre for Biological Material. ${ }^{10}$ The Chinese ESCC cell line (EC109) and HKESC1 were kindly provided by Professor Tsao and Professor Srivastava (University of Hong Kong). All ESCC cell lines were regularly authenticated by checking the morphology and were tested for the absence of mycoplasma contamination (MycoAlert, Lonza, Rockland, ME, USA). Human ESCC samples were collected directly after surgical resection at Queen Mary Hospital (University of Hong Kong, Hong Kong) and Linzhou Cancer Hospital (Henan, China). All samples were immediately frozen in liquid nitrogen and stored at $-80^{\circ} \mathrm{C}$ or fixed in $10 \%$ formalin for paraffin embedding. Samples used in this study were approved by the committees for ethical review of research at the University of Hong Kong and the University of Zhengzhou.

\section{miRCURY LNA array analysis}

Total RNA were extracted from five pairs of ESCC tumour and corresponding non-tumour samples using the mirVana miRNA isolation kit (Ambion, Austin, Texas, USA). Microarray chip analysis was performed and analysed by Exiqon (Vedbaek, Denmark). Before the microarray analysis, RNA quality control was guaranteed by checking the RNA integrity numbers, which is an algorithm measuring the quality of RNA performed by the Agilent 2100 bioanalyzer (Agilent Technologies, Santa Clara, California, USA) at the University of Hong Kong. All the samples had an RNA integrity number greater than 7.0. The fold change was calculated by comparing the expression of miRNA in ESCC tumours pool and normal tissues pool in a $\log _{2}$ format.

\section{Real-time quantitative PCR}

Total RNA was extracted from cultured cells or frozen tissues using TRIzol reagent (Invitrogen, Carlsbad, California, USA) and $2 \mu \mathrm{g}$ RNA was reverse-transcribed using the Advantage
RT-PCR kit (Clontech Laboratories, Inc., Mountain View, California, USA) for first strand complementary DNA synthesis. Quantitative PCR was then carried out with primers for miR-375, SNORD48, 18s rRNA and IGF1R (see supplementary table 1, available online only) with SYBR in the 7900 HT realtime PCR System (Applied Biosystems, Carlsbad, California, USA) following standard quantitative PCR protocol. Briefly, quantitative PCR started with the incubation of samples at $50^{\circ} \mathrm{C}$ for $2 \mathrm{~min}$ followed by $95^{\circ} \mathrm{C}$ for $10 \mathrm{~min}$. Following this, quantitative PCR was repeated for 40 cycles with incubation at $95^{\circ} \mathrm{C}$ for $15 \mathrm{~s}$ followed by at $60^{\circ} \mathrm{C}$ for $1 \mathrm{~min}$. The expression level was normalised against endogenous SNORD48 and 18s rRNA for miR-375 and other tested genes, respectively.

\section{5-Aza-2' -deoxygcitidine treatment}

KYSE30 and KYSE510 $\left(2 \times 10^{5}\right)$ cells were seeded and treated with 5-aza-2'-deoxygcitidine $(50 \mu \mathrm{m}$ and $100 \mu \mathrm{m}$; Sigma-Aldrich Corporation, St Louis, Missouri, USA). Cells were incubated for $72 \mathrm{~h}$ with a change of culture medium every $24 \mathrm{~h}$.

\section{Promoter methylation analysis}

Genomic DNA was extracted and followed by bisulphite treatment and then studied by methylation-specific PCR as previously described. $^{11}$ Bisulphite genomic sequencing (BGS) was also performed for bisulphited DNA. Methylation-specific PCR and BGS primers are listed in supplementary table 1 (available online only).

\section{Tumour-suppressive function of $\mathbf{m i R - 3 7 5}$}

miR-375 precursor and miR-375 inhibitor were purchased from Origene (Rockville, Maryland, USA) and Dharmacon (Lafayette, Colorado, USA), respectively (see supplementary table 2, available online only). According to the clinical characteristics shown in the study performed by Shimada et al, ${ }^{10}$ the tumours from KYSE30 and KYSE510 invaded contiguous structures and thus they may contain invasive properties. Moreover, Shimada et al ${ }^{10}$ confirmed that KYSE30 can form tumour in nude mice. Therefore, miR-375 precursor and miR-375 inhibitor were then stably

Table 1 Differentiated dysregulation of miRNA in ESCC

\begin{tabular}{ll}
\hline miRNA & Fold change \\
\hline Upregulated & \\
hsa-miR-424 & 3.34 \\
hsa-miR-422a & 3.06 \\
hsa-miR-199a-5p & 2.51 \\
hsa-miR-199a-3p/hsa-miR-199b-3p & 2.37 \\
hsa-miR-181a & 2.24 \\
hsa-miR-21 & 2.23 \\
hsa-miR-455-3p & 2.11 \\
hsa-miR-665 & 2.07 \\
hsa-miR-483-5p & 2.04 \\
hsa-miR-127-3p & 2.00 \\
Downregulated & \\
hsa-miR-375 & 5.38 \\
hsa-miR-194 & 3.45 \\
hsa-miR-215 & 3.03 \\
hsa-miR-192 & 2.65 \\
hsa-miR-203 & 2.51 \\
hsa-miR-1249 & 2.29 \\
hsa-miR-29b & 2.06 \\
hsa-miR-1281 & 2.05 \\
hsa-miR-9 & 2.03 \\
hsa-miR-126 & 2.02 \\
hsa-miR-142-5p & 2.01 \\
hsa-miR-1 & 2.01 \\
\hline
\end{tabular}

ESCC, oesophageal squamous cell carcinoma. 
and transiently transfected, respectively, into KYSE30 and KYSE510 cells using Lipofectamine 2000 (Invitrogen). Stable miR-375 overexpressing clones (KYSE30-375+ or KYSE510$375+$ ) and empty vector transfected-cells (KYSE30-vector or KYSE510-vector) were selected by using Geneticin (Invitrogen) at a concentration of $500 \mu \mathrm{g} / \mathrm{ml}$. Individual clones were selected after 3 weeks and the expression was confirmed by quantitative PCR. Transient transfectants miR-375 inhibited clones (KYSE30-375- or KYSE510-375-) and inhibitor negative controls (KYSE30-control and KYSE510-control) were transfected for further study. For foci formation assay, 500, 1000 and 2000 cells (KYSE30-vector, KYSE30-375+, KYSE510-vector or KYSE510-375+) were seeded in six-well plates. Surviving colonies ( $>50$ cells per colony) were counted with Giemsa staining after 7 days in culture. Cell proliferation assay and colony formation in soft agar were carried out as described previously. ${ }^{11}$

For in-vivo study, $2 \times 10^{6}$ miR-375-expressing cells (KYSE30-375 + or KYSE510-375+) and empty vector cells (KYSE30-vector or KYSE510-vector) were injected subcutaneously into the right and left dorsal flank, respectively, of nude mice (five mice per group). Over a 4-week period, tumour formation in nude mice was observed by measuring the tumour volume calculated by the formula $\mathrm{V}=0.5 \times \mathrm{L} \times \mathrm{W}^{2}$. Tumours were then excised and embedded in paraffin for haematoxylin and eoson and immunohistochemistry analysis. All animal experiments were approved by the Committee on the Use of Live Animals in Teaching and Research (CULATR), the University of Hong Kong.

\section{Cell motility and invasion assay}

Wound-healing assay and invasion assay were performed as described previously. ${ }^{11}$ Briefly, for wound-healing assay, wound closure was observed by taking photos under a microscope at 0, 24 and $48 \mathrm{~h}$ after scratching. Invasion was performed with Matrigel (ECM; Chemicon International, Temecula, California, USA) following the manufacturer's instructions. Photographs of three randomly selected fields of the fixed cells were taken and cells were counted. Experiments were repeated three times independently.

\section{Metastasis assay in SCID mice}

Briefly, $2 \times 10^{5}$ cells (four groups including KYSE30-vector, KYSE30-375+, KYSE510-vector and KYSE510-375+) were injected intravenously through the tail vein into 5-6-week-old severe combined immunodeficient (SCID-Beige) mice (five mice per group). After 12 weeks, the number of tumour nodules formed on the liver surfaces was counted. Lungs and livers were excised and embedded in paraffin for further study.

\section{Dual-luciferase reporter assay}

A 301-bp fragment from the 3'-UTR of the IGF1R gene (position 7124-7424) containing the binding sequences of $m i R-375$ was cloned into pGL3 Basic vector (Promega, Madison, Wisconsin, USA). Reverse-sense of the fragments was also cloned by reversing the sequences of the 301-bp fragment. pGL3-Control vector (Promega) was used as a positive control. Stable miR-375 overexpressing clones (KYSE30-375+ and KYSE510-375+) were then
Figure 1 Downregulation of miR-375 in oesophageal squamous cell carcinoma (ESCC), which is associated with poor prognosis. (A) Validation of two most differentially upregulated ( $m i R-424$ and $m i R-422 a$ ) and downregulated (miR-375 and miR-194) miRNAs in tumour (T) and corresponding non-tumour $(\mathrm{N})$ pairs used for microarray analysis. Expression of mir-375 was presented in $\log _{10}$ scale and was normalised against an endogenous control SNORD48. (B) Downregulation of miR-375 was observed in eight ESCC cell lines. SNORD48 was used as an internal control. $N$, pool of non-tumourous tissues. (C) miR-375 was significantly downregulated in 60 primary ESCC from Hong Kong and (D) 45 primary ESCC from Henan compared with their corresponding non-tumour tissues. Expression was shown in $\log _{10}$ scale and was normalised against an endogenous control SNORD48. (E) Downregulation of miR-375 was significantly associated with poorer overall survival and $(F)$ disease-free survival.
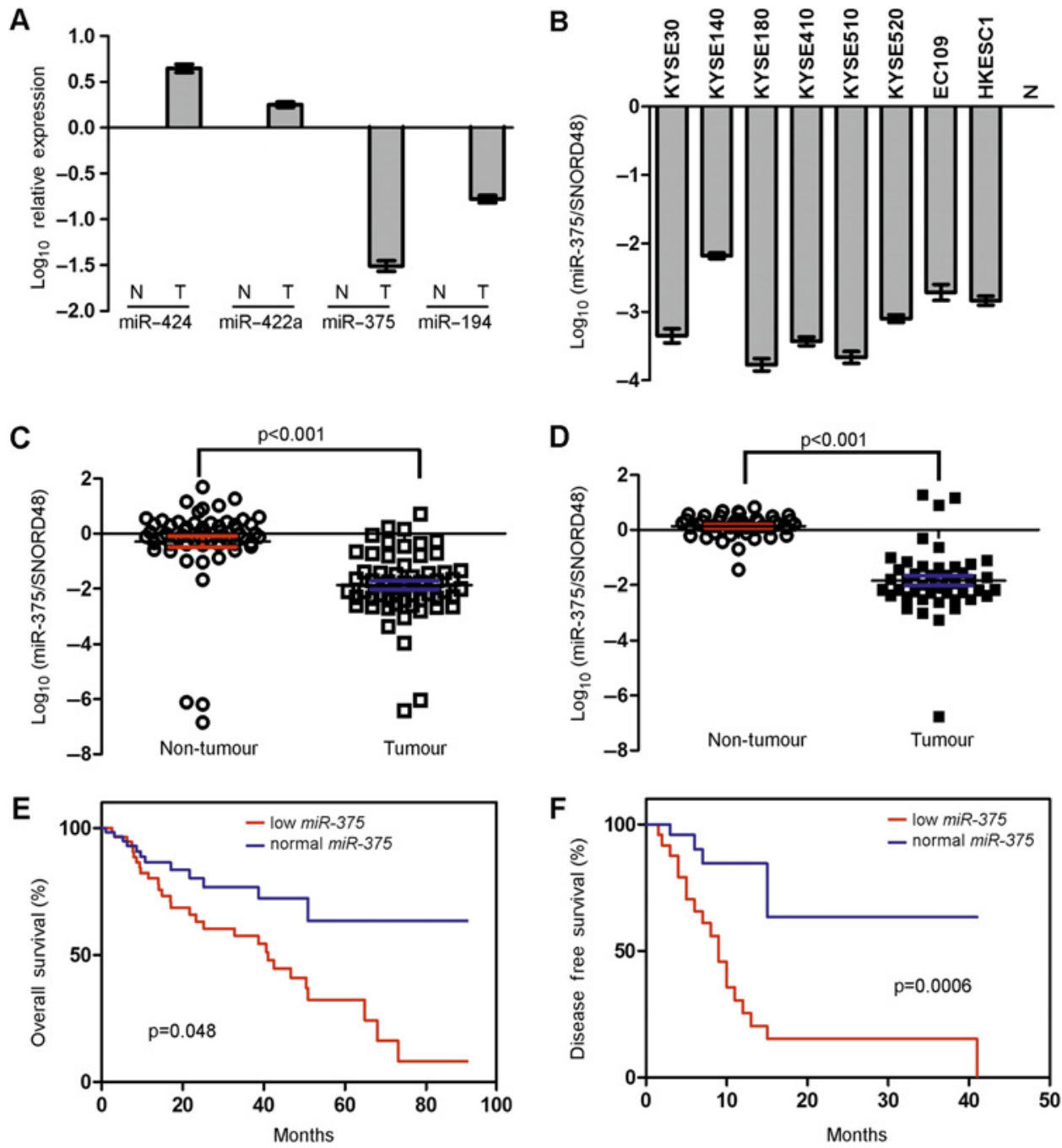
transfected with the reporter constructs containing either the target binding sequence of IGF1R (sense) or the reversed sequence of IGF1R (reverse-sense). Firely and Renilla luciferase signals were determined by the Dual-LUciferase assay kit (Promega).

\section{Western blot analysis}

Western blotting was performed according to the standard protocol with antibodies IGF1R (Novus Biologicals, Littleton, Colorado, USA) and proliferating cell nuclear antigen (PCNA) (Santa Cruz Biotechnology, Santa Cruz, California, USA). $\beta$-Actin (Santa Cruz Biotechnology) was used as the loading control.

\section{Statistical analsysis}

Statistical analysis was carried out using SPSS 16.0 for Windows. Student's t test was used to analyse the results expressed as mean \pm SD. The $\chi^{2}$ test or Fisher's exact test was used to analyse the association of miR-375 expression and clinical-pathological parameters. The survival curves were plotted by using Kaplan-Meier analysis. Differences were considered significant when the $p$ value was less than 0.05

\section{RESULTS}

\section{miRNA profiling in human ESCC}

To investigate the roles of miRNA in ESCC, miRCURY LNA array (Exiqon) containing 850 human miRNA was performed to compare miRNA profiles between ESCC tumours and their corresponding non-tumour tissues pooled from five primary ESCC cases from Hong Kong. Among the 850 miRNA probes, the microarray detected 384 miRNA in our ESCC pairs. With the twofold cut-off point, 22 differentially expressed miRNA (10 upregulated and 12 downregulated) were detected in ESCC tumour tissues compared with their non-tumour counterparts (table 1). The microarray results were validated with quantitative PCR by confirming the expression of two most upregulated (hsa-miR-424 and hsa-miR422a) and downregulated (hsa-miR-375 and hsa-miR-194) miRNA in the same five pairs of human ESCC pool (figure 1A).

\section{miR-375 is frequently downregulated in ESCC}

In this study, hsa-miR-375, which has the largest absolute fold change in the miRCURY LNA array, was further characterised. Quantitative PCR was performed to confirm further the expression of miR-375 in eight ESCC cell lines and 60 pairs of primary ESCC from Hong Kong. Consistent with the miRCURY

Table 2 Association of miR-375 downregulation with clinicopathological features of 60 ESCC patients

\begin{tabular}{lllll}
\hline Clinicopathological & & \multicolumn{2}{l}{ miR-375 expression } & \\
features & Total case (\%) & Negative (\%) & Positive (\%) & p Value \\
\hline Sex & & & & \\
$\quad$ Male & $43(71.67)$ & 76.74 & 23.26 & 0.461 \\
$\quad$ Female & $17(28.33)$ & 82.35 & 17.65 & \\
Age, years & & & & \\
$\quad \leq 66$ & $23(38.33)$ & 78.26 & 21.74 & 0.617 \\
$\quad>66$ & $37(61.67)$ & 78.38 & 21.62 & \\
Clinical Stage & & & & \\
$\quad$ Early stage (I-II) & $22(36.67)$ & 56.45 & 43.55 & \\
$\quad$ Advanced stage (III-IV) & $38(63.33)$ & 92.1 & 7.9 & \\
Distant metastasis & & & & \\
$\quad M_{0}$ & $46(76.67)$ & 86.79 & 13.21 & 0.0001 \\
$\quad M_{1}$ & $14(23.33)$ & 14.29 & 85.71 & \\
Tumour size & & & & \\
$\quad \leq 5 \mathrm{~cm}$ & $21(35)$ & 89.66 & 10.34 & 0.187 \\
$>5 \mathrm{~cm}$ & $39(65)$ & 68.75 & 31.25 & \\
\hline
\end{tabular}

*Significant difference.

ESCC, oesophageal squamous cell carcinoma.

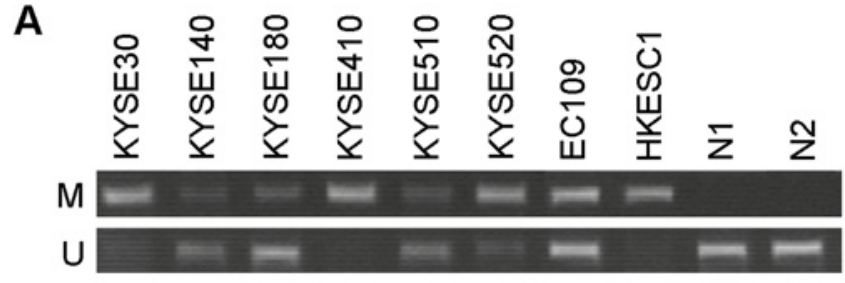

B

bp -580
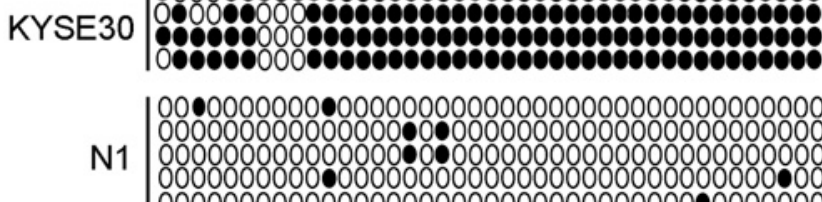
10000000000000000000000000000000000000000

N2

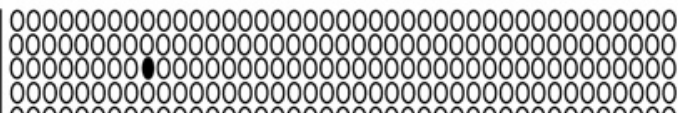

C 00000000000000000000000000000000000000000
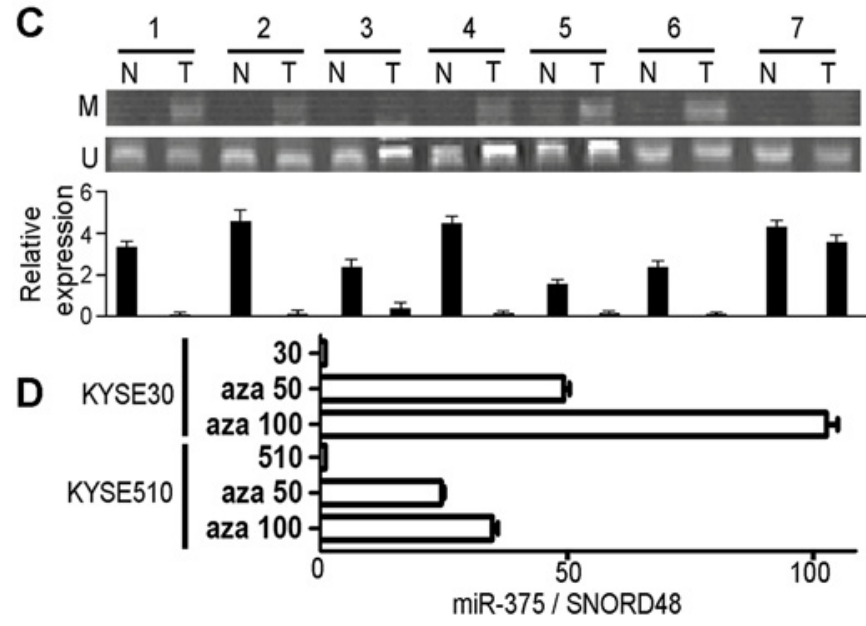

Figure 2 Hypermethylation in promoter region of miR-375 in oesophageal squamous cell carcinoma (ESCC). (A) Methylation-specific PCR (MSP) analysis of miR-375 promoter region in eight ESCC cell lines. Methylated allele was detected in all ESCC cell lines while only unmethylated allele was detected in $\mathrm{N} 1$ and $\mathrm{N} 2 . \mathrm{M}$, methylated allele; $\mathrm{U}$, unmethylated allele; N1, pool of non-tumour tissues from five ESCC; N2, pool of blood lymphocytes from five healthy donors. (B) Bisulphite genomic sequencing (BGS) of miR-375 promoter region in ESCC cell line KYSE30, N1 and N2 (five clones for each sample). Each vertical bar represents a $\mathrm{CpG}$ site. High densities of methylated $\mathrm{CpG}$ sites were observed in KYSE30 but not in N1 and N2. Black and white circles represent methylated and unmethylated $\mathrm{CpG}$ sites, respectively. (C) Representative of methylation-specific PCR results of miR-375 in primary ESCC. Methylation of miR-375 was detected in more than half $(57.8 \%)$ of the tumour specimens (upper panel). $\mathrm{M}$, methylated allele; $\mathrm{U}$, unmethylated allele. The relative expression of miR-375 for each sample was shown in the lower panel. SNORD48 was used as an internal control. (D) Demethylation treatment with 5-aza-2' -deoxycytidine (Aza) could restore miR-375 expression in KYSE30 and KYSE510 cells. SNORD48 was used as an internal control. 30 and 510, ESCC cell lines KYSE30 and KYSE510 without treatment; aza 50, cell lines treated with $50 \mu \mathrm{M}$ 5-aza-2' -deoxycytidine; aza 100, cell lines treated with $10 \mu \mathrm{M}$ 5-aza-2' -deoxycytidine. 
Figure 3 miR-375 has a strong tumour-suppressive effect. (A) Relative expression of miR-375 was detected by quantitative PCR in stably transfected KYSE30 (KYSE30-375+) and KYSE510 (KYSE510-375+) cells relative to empty vectors (KYSE30-vector or KYSE510vector). Expression was normalised against an endogenous control SNORD48. (B) miR-375 could inhibit the foci formation ability of KYSE510-375+ cells. The results are expressed as mean $\pm S D$ of three independent experiments. Vec, empty vector KYSE510-vector; miR, stably transfected clone KYSE510-miR375+. (C) Colony formation in soft agar was significantly inhibited by miR-375. The results are expressed as mean $\pm S D$ of three independent experiments. (D) Cell growth rates were suppressed by miR375 in KYSE30-375 + (left) and KYSE510$375+$ (right) detected by XTT assay. The results are expressed as mean $\pm S D$ of three independent experiments.

${ }^{*} p<0.05$. (E) Tumour growth curves show that miR-375 could inhibit tumour formation in nude mice induced by KYSE30-375 + (left) and miR510 (right) cells. Points and bars represent the mean of five mice with SD. ${ }^{*} p<0.05$. For the representatives of tumour formed in nude mice, tumours were induced by empty vector-transfected cells (left dorsal flanks, indicated by arrows) and miR-375-transfected cells (right dorsal flanks), respectively.
A

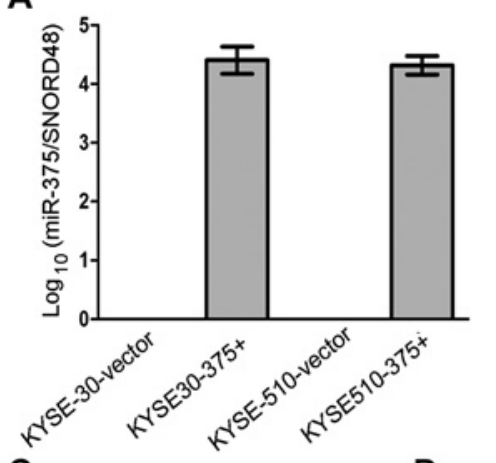

B

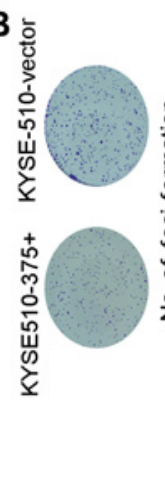

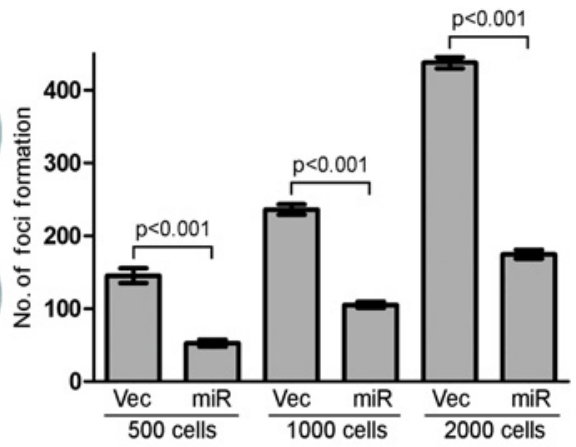

C
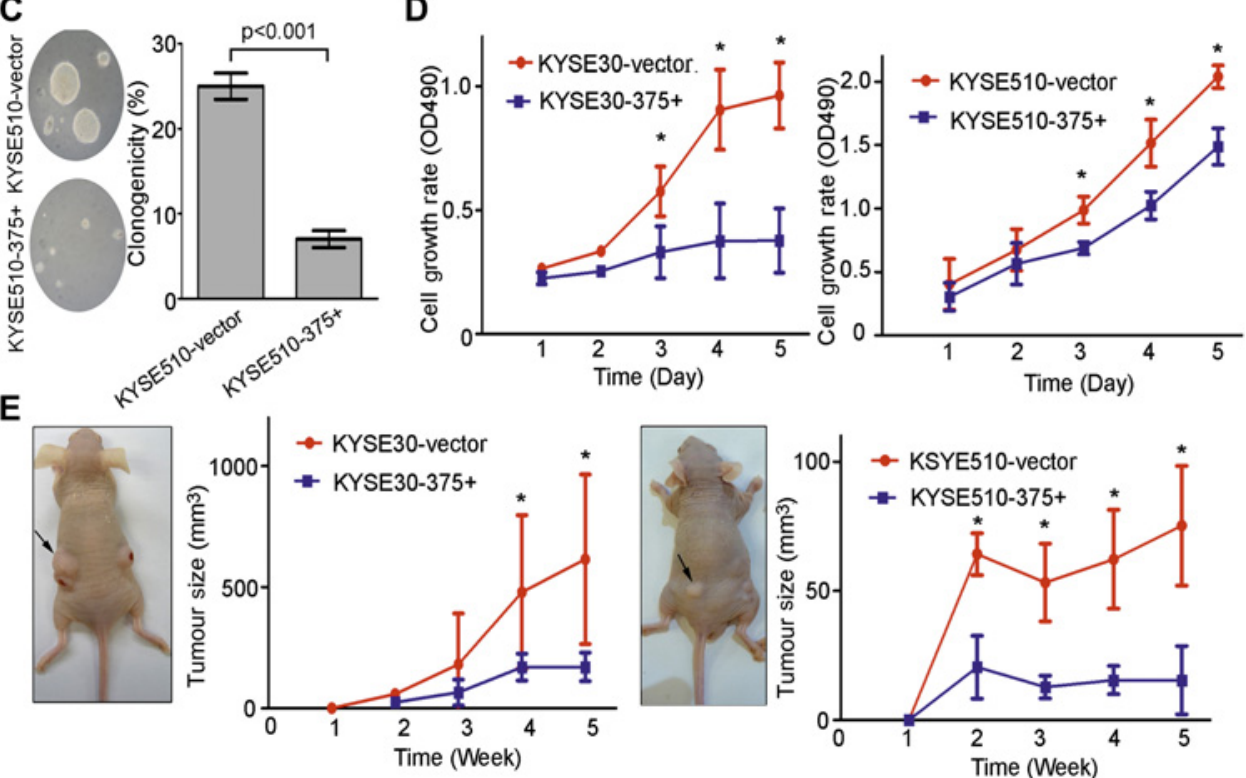

LNA array, miR-375 was strongly downregulated in ESCC cell lines and primary ESCC samples from Hong Kong (figure 1B,C). As Henan province is one of the ESCC high-risk areas in the world, the expression of miR-375 was also investigated in 45 pairs of primary ESCC and corresponding non-tumour tissues from Henan. Results showed that miR-375 was also significantly downregulated in Henan ESCC samples (figure 1D).

\section{Downregulation of $m i R-375$ is associated with poor prognosis of ESCC}

To address the clinical significance of the downregulation of $m i R-375$ in ESCC, the correlation of miR-375 downregulation with clinical-pathological features from 60 Hong Kong patients was investigated. The results showed that the downregulation of miR-375 was significantly correlated with advanced clinical stage and distant metastasis while no significant correlation was observed in other clinical-pathological parameters (table 2). To investigate the prognostic significance of miR-375 in ESCC, Kaplan-Meier analysis was performed illustrating that low miR-375 expression was associated with poorer overall survival and disease-free survival in the ESCC cohort (figure 1E,F). These results suggested that the downregulation of miR-375 may play an important role in the development and progression of ESCC.

\section{Downregulation of $\mathbf{m i R}-375$ is caused by promoter hypermethylation}

To understand the mechanism of miR-375 downregulation in ESCC, the methylation status in the promoter region of miR-375 was tested. A previous report has indicated that the $-768 \mathrm{bp}$ upstream of pre-miR-375 region contains promoter function. ${ }^{12}$ In the present study, the promoter region was characterised by methylation-specific PCR using methylation or unmethylationspecific primers. In each of the eight ESCC cell lines in which the downregulation of miR-375 was investigated, at least one allele was methylated (figure 2A). In contrast, only the unmethylated allele was detected in a pool of non-tumourous tissues (N1) and a pool of peripheral blood lymphocytes from healthy donors (N2) (figure 2A). In addition, BGS was used to characterise the methylation details of miR-375 promoter in KYSE30, N1 and N2. High densities of methylated CpG sites were only observed in KYSE30 but not in N1 and N2 (figure 2B). To confirm further that the promoter hypermethylation of $m i R-375$ was the major mechanism causing the downregulation of $m i R-375,45$ pairs of primary ESCC tumours and the corresponding non-tumour tissues from Henan were analysed by methylation-specific PCR. Methylation of miR-375 was detected in 26 of 45 $(57.8 \%)$ tumour specimens. The frequency of methylation was noticeably higher in ESCC with miR-375 downregulation $(19 / 31,61.3 \%)$ than that in ESCC with normal miR-375 expression $(7 / 14,50 \%$ ) (figure $2 \mathrm{C})$. To validate the result further, KYSE30 and KYSE510 cells were treated with the demethylating agent 5-aza-2'-deoxycytidine. After the treatment, the expression of miR-375 was restored (figure 2D), indicating that promoter hypermethylation was the major mechanism of the downregulation of $\mathrm{miR}-375$.

\section{miR-375 suppresses tumour cell growth}

To explore the potential tumour-suppressive role of $m i R-375$ in ESCC, $m i R-375$ precursor was cloned and stably transfected into 
Figure 4 miR-375 suppresses tumour metastasis. (A) Wound-healing assay shows that cell motility could be effectively suppressed by KYSE30-375 + (left) and KYSE510-375+ (right). Representative images were taken at time 0,24 and $48 \mathrm{~h}$ after scratching. (B) miR-375 could successfully impede tumour cell invasion in KYSE30 (left) and KYSE510 (right) cells $(p<0.001)$. The results are expressed as mean $\pm S D$ of three independent experiments. (C) miR-375 could suppress oesophageal squamous cell carcinoma (ESCC) metastasis in vivo. Representative livers derived from severe combined immunodeficient (SCID) mice in KYSE30 (left) and KYSE510 (right) are shown. The formation of metastatic nodules at the liver surface could be significantly suppressed by KYSE30-375+ and KYSE510-375+. (D) Metastatic tumours in lung could be induced by empty vector-transfected cells (KYSE30-vector and KYSE510-vector) in SCID mice, but not by miR-375transfected cells (KYSE30-375+ and KYSE510-375+).
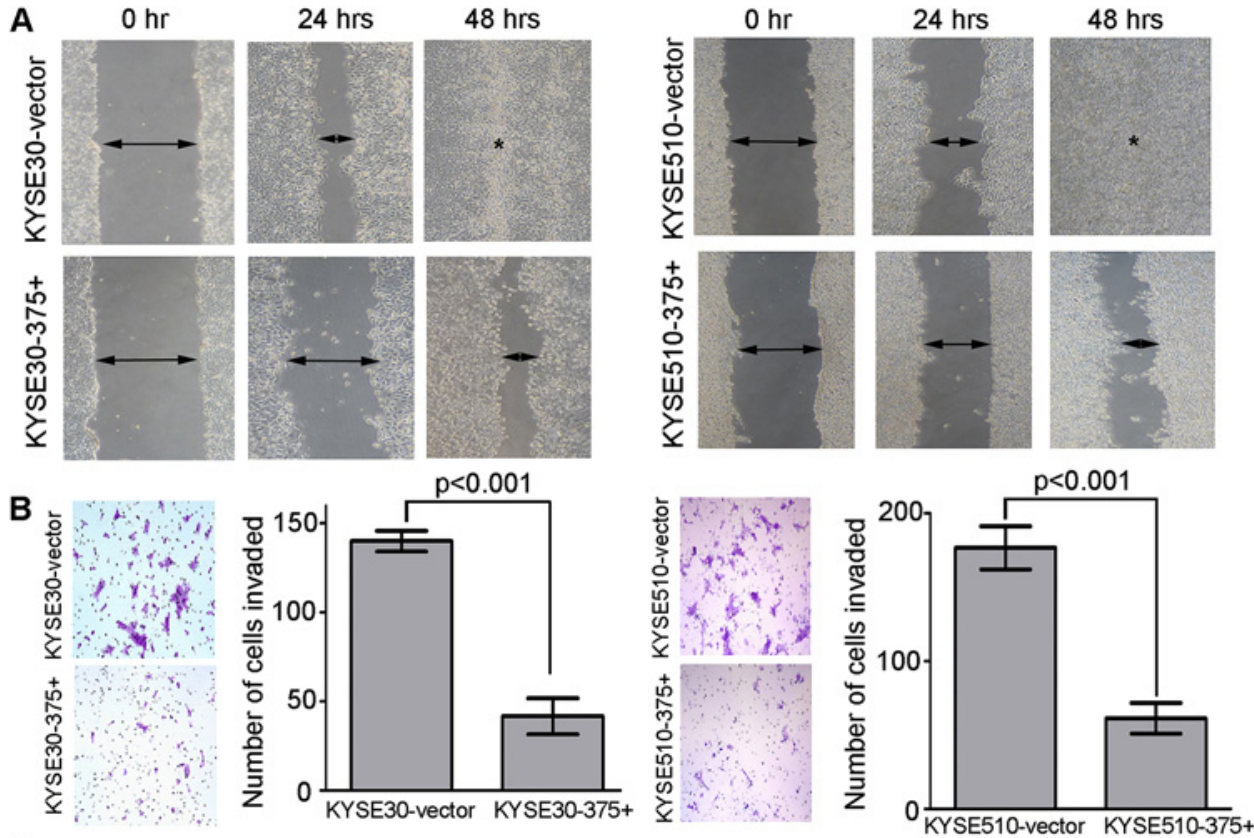

C
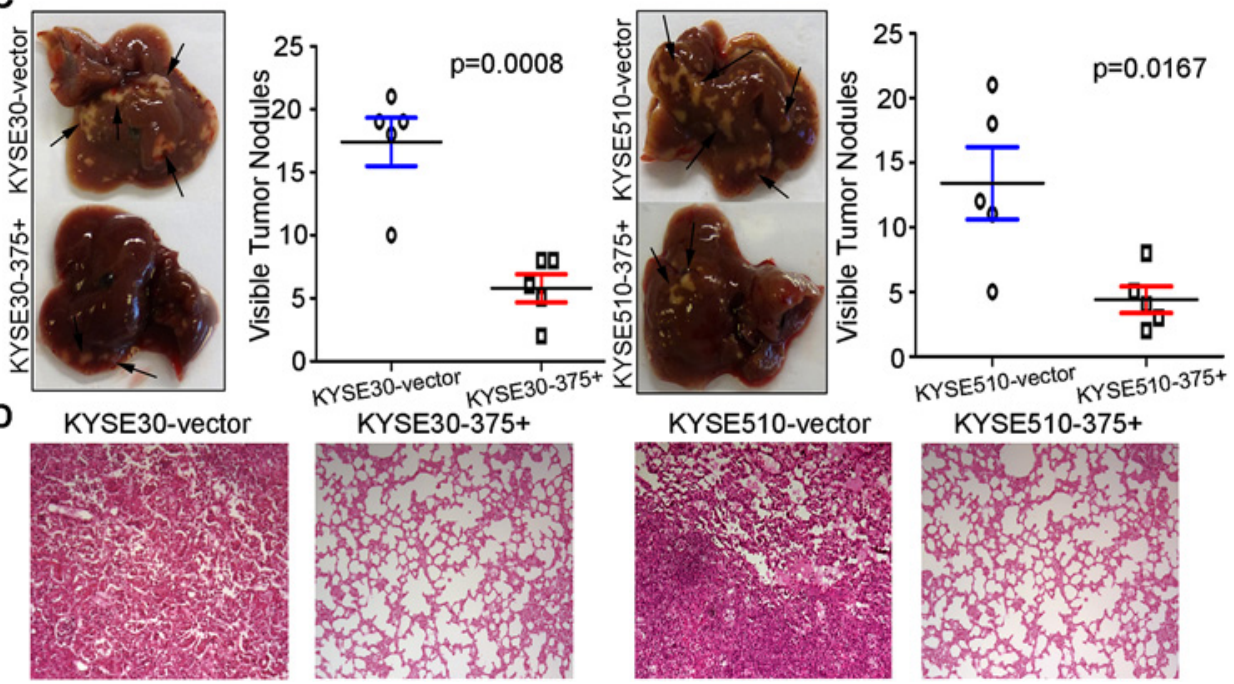

ESCC cell lines KYSE30 and KYSE510. The expression of mature miR-375 in stable transfectants was confirmed by quantitative PCR (figure 3A). Functional study showed that miR-375 could suppress the foci formation (figure $3 \mathrm{~B}$ ) and colony formation in soft agar (figure 3C) compared with empty vector-transfected cells. XTT assay also showed that miR-375 could inhibit cell growth rate (figure $3 \mathrm{D}$ ). In addition, tumour formation in nude mice was performed by injecting KYSE30-375+ cells $(n=5)$ or KYSE510-375+ cells ( $n=5)$, while KYSE30-vector or KYSE510vector cells were used as empty vector controls. Consistent with in-vitro results, $m i R-375$ could inhibit tumour formation in nude mice (figure $3 \mathrm{E}$ ). To detect the proliferating cells, immunohistochemical staining of PCNA was performed. As expected, less PCNA expression was observed in the KYSE30-375+ and KYSE510-375+-derived tumours compared with KYSE30-vector and KYSE510-vector-derived tumours (see supplementary figure 1 , available online only). These results implied that miR-375 has a strong ability to suppress tumour cell growth.

\section{miR-375 suppresses ESCC invasion and metastasis}

In addition to cell growth inhibition, the effect of miR-375 on tumour invasion and metastasis was also addressed in this study.
Wound-healing assay showed that miR-375 could dramatically suppress tumour cell mobility in KYSE30-375+ and KYSE510$375+$ cells compared with the empty vector-transfected cells (figure 4A). To validate that mobility suppression is not caused by the inhibitory effect of tumour cell growth, invasion assay was also performed. The result demonstrated that miR-375 could suppress the invasive ability of ESCC cells (figure 4B). To investigate further the suppressive effect of $m i R-375$ on tumour metastasis, an experimental metastasis animal model was performed by injecting KYSE30-375+ or KYSE510-375+ cells into the tail vain of SCID mice (five mice per group). Empty vector-transfected (KYSE30-vector or KYSE510-vector) cells were used as controls (five mice per group). The mice were killed and metastatic nodules were counted on the surface of liver after 12 weeks. The numbers of nodules formed in the liver induced by KYSE30-375+ and KYSE510-375+ cells were significantly more reduced than those induced by KYSE30-vector and KYSE510-vector cells (figure 4C). Although no visible metastatic nodule was observed on the surface of the lung, metastatic lesions were detected in lung by haematoxylin and eosin staining (figure 4D). These results indicated that miR-375 could effectively suppress tumour metastasis in vitro and in vivo. 
Figure 5 Insulin-like growth factor 1 receptor (IGF1R) is a direct downstream target of miR-375. (A) The predicted binding sequence of human hsa-miR-375 and its binding site in the $3^{\prime}$-untranslated region (UTR) of IGF1R (sense) were presented for alignment. Reverse-sense was the reverse sequences of 3 ' -UTR of IGF1R without the binding sites of miR375. (B) Quantitative PCR result shows that IGF1R could be downregulated by miR-375 in KYSE30-375+ (left) and KYSE510-375 + (right) cells ( $p<0.001$ ). Expression was normalised against an endogenous control 18s rRNA and results are expressed as mean $\pm S D$ of three independent experiments. (C) Western blot analysis shows that miR375 could inhibit the protein expression of IGF1R in KYSE30-375 + (left) and KYSE510-375 + (right) cells. $\beta$-Actin was used as an internal control. (D) The expression of IGF1R was negatively correlated with the miR-375 expression level in eight oesophageal squamous cell carcinoma (ESCC) cell lines. Expression was shown in $\log _{10} .18 \mathrm{~s}$ rRNA and SNORD48 were used as internal controls for IGF1R and miR-375 expression, respectively. (E) Luciferase assay was used to confirm the interaction of miR375 with IGF1R. 3 '-UTR of IGF1R containing the target binding site (sense) and the reverse sequences of 3 -UTR of IGF1R (reverse-sense) were cloned into downstream of a firefly luciferase gene. The plasmids (sense or reverse-sense) were transfected into empty vector and miR-375 stably expressing cells (miR-

375). Renilla luciferase plasmid was

co-transfected for normalisation. pGL3-Control vector was co-transfected as positive control. Control, positive control; +, plasmid added; -, no plasmid added. ${ }^{*} p<0.05$. (F) Expression level of IGF1R was tested in 60 primary ESCC from Hong Kong by quantitative PCR. Results showed that IGF1R was frequently upregulated in tumour tissues (T) compared with their non-tumour counterparts (N) (left). 18s rRNA was used as an internal control. A negative correlation between IGF1R and miR-375 expressions in 60 ESCC pairs from Hong Kong was determined with linear regression lines and Pearson correlation significance $(p=0.008$, Pearson correlation -0.25$)$ (right).

\section{IGF1R is the potential target of miR-375}

As miR-375 inhibited tumour cell growth and metastasis, we would like to investigate the potential target of $m i R-375$. By searching the potential targets of miR-375 with TargetScan, pictar and miRanda, we compiled all the predicted genes for functional clustering analysis classified by DAVID 6.7, Kegg and Panther databases. ${ }^{13}$ Based on the method previously used by other studies, ${ }^{14}{ }^{15}$ IGF1R was identified as the target of miR375 , which contains putative target sequence (figure $5 \mathrm{~A}$ ). To validate the in-silico prediction, quantitative PCR and western blotting were used to test whether the expression of IGF1R could be repressed by $m i R-375$. The result showed that miR-375 could downregulate IGF1R expression in both the mRNA level (figure 5B) and protein level (figure 5C). Interestingly, mRNA expression of IGF1R was negatively correlated with miR-375 in ESCC cell lines (figure 5D). To validate whether IGF1R is the direct downstream target of $m i R-375$, a fragment of the $3^{\prime}$-UTR of IGF1R (301 bp) containing the potential miR-375 binding site was cloned into a vector with the firefly luciferase reporter gene. Luciferase activity was reduced by approximately $30 \%$ compared with the control (figure $5 \mathrm{E}$ ), which could be reversed by treating culture cells with the antisense IGF1R 3 '-UTR (figure 5E). This ensured the specificity of binding between miR-375 and the $3^{\prime}$-UTR of IGF1R. To investigate the correlation between miR-375 and IGF1R in clinical specimens, quantitative PCR was performed to detect expressions of miR-375 and IGF1R in 60 primary ESCC samples from Hong Kong. The expression of IGF1R was significantly higher in ESCC tumour, compared with the corresponding non-tumour tissue (figure 5F). Moreover, a significant negative correlation was observed between $m i R-375$ and IGF1R (figure $5 \mathrm{~F}$ ). In addition, immunohistochemical staining was applied to compare IGF1R expression in tumours derived from nude mice injected with KYSE30-vector or KYSE30-375+ using anti-IGF1R antibody. The result showed that the expression of IGF1R was repressed in KYSE30-375+-generated tumours, compared with KYSE30vector-generated tumours (see supplementary figure 2 , available online only), suggesting that the tumour-suppressive effect of $m i R-375$ was via suppressing IGF1R expression.

Knockdown of miR-375 increases tumour growth and metastasis To confirm further the tumour-suppressive function of miR-375, $m i R-375$ was hindered by adding the specific inhibitor of $m i R-375$ into the culture cells. As miR-375 was downregulated in all 
Figure 6 Knockdown of miR-375 enhanced tumour growth and metastasis. (A) miR-375 inhibitor was transiently transfected into KYSE30 and KYSE510 using lipofectamine. Inhibition was confirmed by quantitative PCR in KYSE30-375 - (left) and KYSE510-375(right). SNORD48 was used as an internal control. ${ }^{*} p<0.05$ (B) The expression of insulin-like growth factor 1 receptor (IGF1R) was increased after knockdown of miR-375. ${ }^{*} \mathrm{p}<0.05$. 18s rRNA was used as an internal control. (C) XTT assay showed that the inhibition of miR-375 increased oesophageal squamous cell carcinoma (ESCC) cell growth. ${ }^{*} p<0.05$. (D) The efficiency of foci formation was enhanced after the suppression of miR$375 .{ }^{*} \mathrm{p}<0.05$. (E) Tumour cell motility was enhanced after the repression of miR-375. Representative images were taken at time $0 \mathrm{~h}$ and $24 \mathrm{~h}$ after scratching. (F) Cell invasive ability was improved after the inhibition of $m i R-375$. The results are expressed as mean $\pm S D$ of three independent experiments. ${ }^{*} p<0.05$.
A
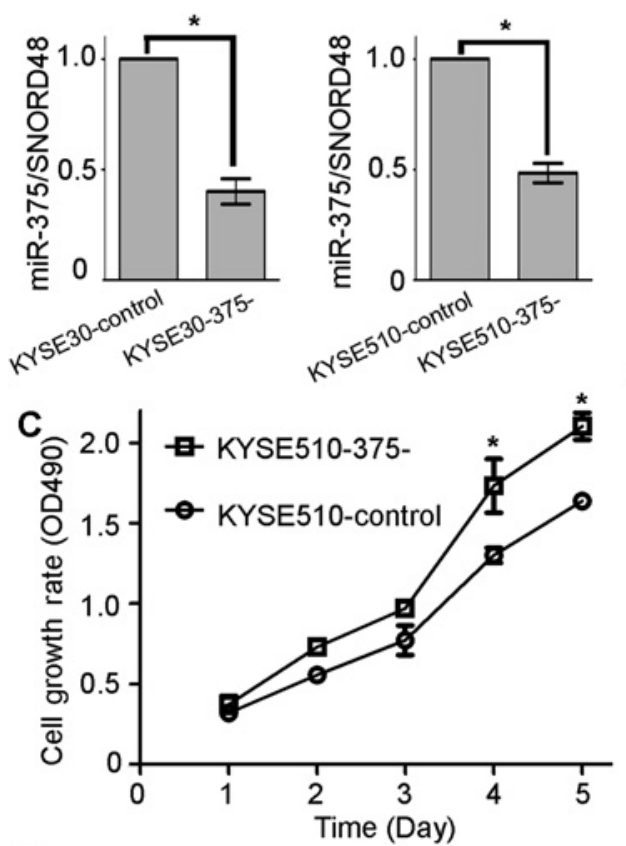

E
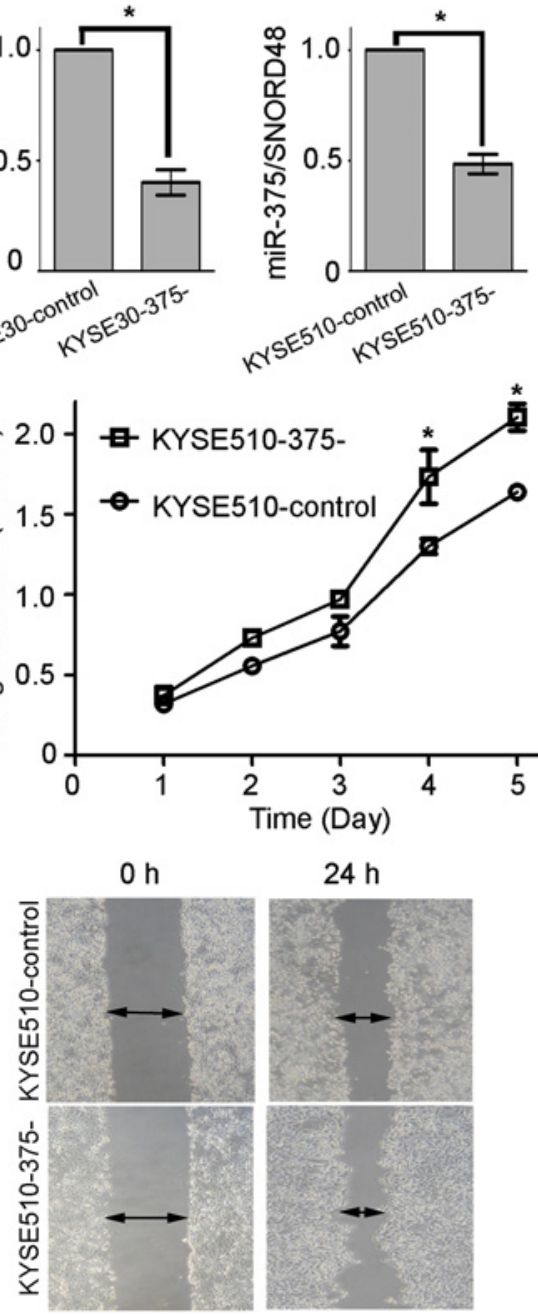

B
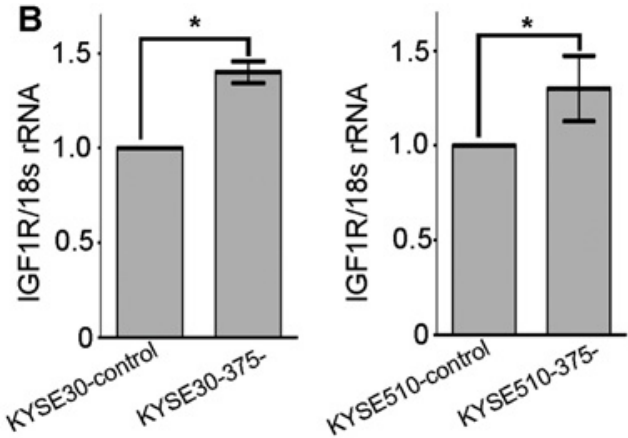

D

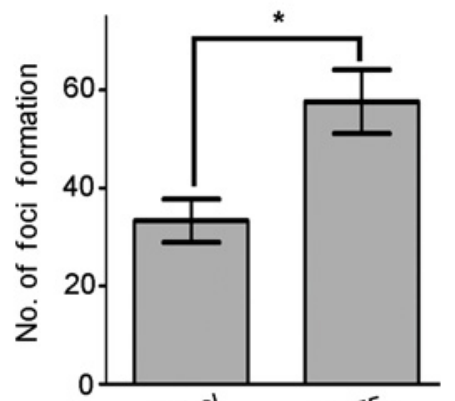

$\mathbf{F}$ KYSE510-control KYSE510-375-

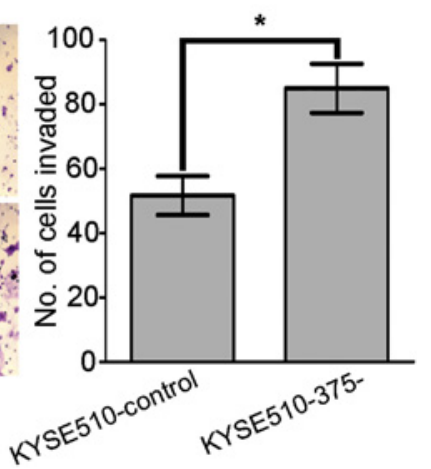

tested ESCC cell lines, miR-375 was knocked down in miR-375expressing clones. The silencing effect of $m i R-375$ was confirmed by quantitative PCR (figure $6 \mathrm{~A}$ ). Moreover, the expression of IGF1R was restored after the $m i R-375$ was inhibited (figure $6 \mathrm{~B}$ ). XTT and foci formation assays also indicated that knockdown of miR-375 could enhance the cell growth rate and increase foci formation (figure 6C,D). In addition, tumour mobility and invasive ability were also enhanced (figure 6E,F). Taken together, these results indicated that tumour growth and metastasis was enhanced after the knockdown of miR-375.

\section{DISCUSSION}

miRNA have been reported to play important roles in cancer development and progression through repressing downstream target genes. ${ }^{16}$ The first evidence to link miRNA and cancer came from B-cell chronic lymphocytic leukaemia studies. ${ }^{17}$ Since then, abnormal expressions of miRNA have been detected in different types of cancers. ${ }^{18-20}$ However, little is known about the dysregulation of miRNA in ESCC. For a better understanding of the molecular mechanisms involved in the development and progression of ESCC, miRNA profiles between tumour and corresponding non-tumour tissues from five primary ESCC cases were compared. Twenty-two differentially expressed miRNA were detected. Following this, we validated two most upregulated (miR-424 and miR-422a) and two downregulated (miR-375 and miR-194) miRNA.
All of them have been associated with human cancers. For example, miR-424 was upregulated in several cancers such as colorectal cancer, tongue cancer, chronic myelogenous leukaemia and renal cell carcinomas. ${ }^{21-24}$ One recent study showed that miR$422 a$ could be used as a diagnostic marker for osteosarcoma. ${ }^{25}$ The role of miR-194 in cancer development is complicated. For instance, some studies demonstrated that it is downregulated in endometrioid endometrial cancer and ESCC. ${ }^{26} 27$ However, different groups showed that miR-194 was upregulated in oesophageal adenocarcinoma and prostate cancer. ${ }^{28} 29$ Due to the largest absolute fold change, $m i R-375$ was chosen for further study. $m i R-375$ has been reported to be important in the regulation of insulin secretion. ${ }^{30}$ One study found that miR-375 is able to suppress glucose-induced insulin secretion with no change in the glucose-mediated production of ATP or intracellular calcium. ${ }^{31}$ The downregulation of miR-375 has been reported in gastric cancer, ${ }^{32}$ hepatocellular carcinoma ${ }^{33}$ and head and neck cancer. ${ }^{34}$ However, the function of miR-375 in ESCC remains unclear.

In the present study, we found that miR-375 was frequently downregulated in primary ESCC cases from Hong Kong $(n=60)$ and Henan $(n=45)$. Interestingly, the expression patterns of miR-375 in both groups are very similar. Therefore, we assumed that miR-375 plays a similar tumour-suppressing role in ESCC development in both populations. The downregulation miR-375 was significantly associated with advanced clinical stage, distant 
metastasis, poorer overall survival and poorer disease-free survival in ESCC. Further study found that the mechanism of miR-375 downregulation was closely associated with the hypermethylation in its promoter region. The survival rate of ESCC patients remains unsatisfactory despite advanced treatments. ${ }^{35}$ Therefore, there is a great need to identify biomarkers for the prediction of ESCC prognosis and to develop novel therapeutic drugs for the improvement of ESCC patients' survival. As the downregulation of miR-375 was correlated with poorer survival of ESCC, miR-375 might be used as a prognostic biomarker in ESCC.

In this study, the effects of miR-375 on ESCC development and progression were also investigated by both in-vitro and invivo assays. By transfecting miR-375 into ESCC cell lines (KYSE30 and KYSE510), the expression of miR-375 could effectively inhibit tumour cell growth rate, foci formation, colony formation in soft agar and tumour formation in nude mice. Furthermore, the expression of miR-375 could suppress tumour cell motility and invasion. A metastasis experimental animal model also confirmed that miR-375 could reduce the formation of metastatic tumour nodules in liver and lung.

Each miRNA can potentially downregulate many target genes through binding their $3^{\prime}-U T R$. As the exact binding of miRNA and target genes depend on cell types and microenvironment, miRNA in different tissues or cancers may play different roles. ${ }^{17}$ One important task is to identify the downstream target genes regulated by the dysregulated miRNA. By using DAVID Bioinformatics clustering tool (http://david.abcc.ncifcrf.gov/), ${ }^{13}$ one candidate downstream target gene of miR-375, IGF1R, was identified. IGF1R is frequently overexpressed in many malignancies, and plays a crucial role in promoting cell proliferation, survival and metastasis. ${ }^{36}$ IGF1R has been shown to bind to the regulatory subunit of phosphatidylinositol 3-kinase, PIK3R3, through the Src homology 2 (SH2) domain and activate the phosphatidylinositol 3-kinase pathway. ${ }^{37}$ In the present study, quantitative PCR and western blot analysis demonstrated that miR-375 could decrease the expression of IGF1R in both mRNA and protein levels. In addition, inhibiting miR-375 expression could increase IGF1R expression. Luciferase assay showed that miR-375 could interact with the 3'-UTR of IGF1R. Moreover, the expression of IGF1R was negatively correlated with miR-375 in clinical ESCC specimens. These data strongly suggest that the tumour-suppressive function of $m i R-375$ is through the downregulation of IGF1R, which may have a strong implication on the Akt pathway.

The identification and characterisation of the tumoursuppressing effect of miR-375 may provide a novel therapeutic target. A recent study showed that the delivery of tumoursuppressive miRNA in mice could effectively suppress tumourigenesis in liver cancer without causing toxicity, ${ }^{38}$ indicating that a tumour-suppressive miRNA can be used as an attractive approach for cancer treatment. In summary, we demonstrated in this study that miR-375 is frequently downregulated in ESCC tumour cells, which is significantly associated with poorer prognosis. The tumour-suppressing function of miR375 is through repressing its downstream target gene IGF1R, and downregulation of $m i R-375$ is one of the molecular mechanisms leading to the development and progression of ESCC.

Acknowledgements The authors would like to thank Dr Stephanie KY MA, the Department of Pathology, the University of Hong Kong for her precious advice.

Funding This work was supported by grants from Hong Kong Research Grant Council Central Allocation (HKUST 2/06C); the National Natural Science Foundation of China (30700820, 30772475 and 30971606); and Sun Yat-Sen University 'Hundred Talents Program' (85000-3171311).

\section{Competing interests None.}

Ethics approval Samples used in this study were approved by the committees for ethical review of research at the University of Hong Kong and the University of Zhengzhou.

Provenance and peer review Not commissioned; externally peer reviewed.

\section{REFERENCES}

1. Enzinger PC, Mayer RJ. Esophageal cancer. N Engl J Med 2003;349:2241-52.

2. Li JY. Epidemiology of esophageal cancer in China. Natl Cancer Inst Monogr 1982;162:113-20.

3. Risk JM, Mills HS, Garde J, et al. The tylosis esophageal cancer (TOC) locus: more than just a familial cancer gene. Dis Esophagus 1999:12:173-6.

4. Ries LAG, Eisner MP, Kosary C, et al. SEER Cancer Statistics Review, 1973-1999. Bethesda, MD: National Cancer Institute, 2002.

5. Cho WC. OncomiRs: the discovery and progress of microRNAs in cancers. Mol Cancer 2007;6:60.

6. Iorio MV, Croce CM. MicroRNAs in cancer: small molecules with a huge impact. $J$ Clin Oncol 2009;27:5848-56.

7. Rosenfeld N, Aharonov R, Meiri E, et al. MicroRNAs accurately identify cancer tissue origin. Nat Biotechnol 2008;26:462-9.

8. Hiyoshi Y, Kamohara $\mathrm{H}$, Karashima $\mathrm{R}$, et al. Micro-RNA-21 regulates the proliferation and invasion in esophageal squamous cell carcinoma. Clin Cancer Res 2009:15:1915-22.

9. Feber A, Xi L, Luketich JD, et al. MicroRNA expression profiles of esophageal cancer. J Thorac Cardiovasc Surg 2008;135:255-60.

10. Shimada Y, Imamura M, Wagata $\mathrm{T}$, et al. Characterization of 21 newly established esophageal cancer cell lines. Cancer 1992;69:277-84.

11. Kong KL, Kwong DL, Fu L, et al. Characterization of a candidate tumor suppresso gene uroplakin $1 \mathrm{~A}$ in esophageal squamous cell carcinoma. Cancer Res 2010;70:8832-41.

12. Avnit-Sagi T, Kantorovich L, Kredo-Russo S, et al. The promoter of the pri-miR-375 gene directs expression selectively to the endocrine pancreas. PLoS One 2009;4: e5033.

13. Huang DW, Sherman BT, Lempicki RA, et al. Systematic and integrative analysis of large gene lists using DAVID bioinformatics resources. Nat Protoc 2009;4:44-57.

14. Laganà $\mathbf{A}$, Forte $\mathrm{S}$, Russo $\mathrm{F}$, et al. Prediction of human targets for viral-encoded microRNAs by thermodynamics and empirical constraints. J RNAi Gene Silencing 2010;6:379-85.

15. Veksler-Lublinsky I, Shemer-Avni Y, Kedem K, et al. Gene bi-targeting by viral and human miRNAs. BMC Bioinformatics 2010;11:249.

16. Croce $\mathbf{C M}$. Causes and consequences of microRNA dysregulation in cancer. Nat Rev Genet 2009;10:704-14.

17. Calin GA, Dumitru CD, Shimizu M, et al. Frequent deletions and down-regulation of micro- RNA genes miR15 and miR16 at 13q14 in chronic lymphocytic leukemia. Proc Natl Acad Sci U S A 2002:99:15524-9.

18. Chen CZ. MicroRNAs as oncogenes and tumor suppressors. N Engl J Med 2005:353:1768-71

19. Hammond SM. MicroRNAs as oncogenes. Curr Opin Genet Dev 2006;16:4-9.

20. Esquela-Kerscher A, Slack FJ. OncomirsmicroRNAs with a role in cancer. Nature Rev Cancer 2006:6:259-69.

21. Wang X, Wang $\mathrm{J}, \mathrm{Ma} \mathrm{H}$, et al. Downregulation of miR-195 correlates with lymph node metastasis and poor prognosis in colorectal cancer. Med Oncol 2011, Epub ahead of print.

22. Rentoft M, Fahlén J, Coates PJ, et al. miRNA analysis of formalin-fixed squamous cell carcinomas of the tongue is affected by age of the samples. Int J Oncol 2011;38:61-9.

23. Hussein K, Büsche G, Muth $M$, et al. Expression of myelopoiesis-associated microRNA in bone marrow cells of atypical chronic myeloid leukaemia and chronic myelomonocytic leukaemia. Ann Hematol 2011;90:307-13.

24. Petillo D, Kort EJ, Anema J, et al. MicroRNA profiling of human kidney cancer subtypes. Int J Oncol 2009:35:109-14.

25. Gougelet A, Pissaloux D, Besse A, et al. Micro-RNA profiles in osteosarcoma as a predictive tool for ifosfamide response. Int $J$ Cancer 2010;129:680-90.

26. Chung TK, Lau TS, Cheung TH, et al. Dysregulation of microRNA-204 mediates migration and invasion of endometrial cancer by regulating FOXC1. Int $J$ Cancer. Published Online First: 11 March 2011. doi:10.1002/ijc.26060.

27. Wong TS, Liu XB, Wong BY, et al. Mature miR-184 as potential oncogenic microrna of squamous cell carcinoma of tongue. Clin Cancer Res 2008;14:2588-92.

28. Wijnhoven BP, Hussey DJ, Watson DI, et al. MicroRNA profiling of Barrett's oesophagus and oesophageal adenocarcinoma. Br J Surg 2010;97:853-61.

29. Tong AW, Fulgham $\mathrm{P}$, Jay $\mathrm{C}$, et al. MicroRNA profile analysis of human prostate cancers. Cancer Gene Ther 2009:16:206-16.

30. Poy MN, Hausser J, Trajkovski M, et al. miR-375 maintains normal pancreatic \{alpha\}- and \{beta\}-cell mass. Proc Natl Acad Sci U S A 2009;106:5813-28.

31. Poy MN, Eliasson L, Krutzfeldt J, et al. A pancreatic islet-specific microRNA regulates insulin secretion. Nature 2004;432:226-30.

32. Tsukamoto $\mathbf{Y}$, Nakada C, Noguchi T, et al. MicroRNA-375 is downregulated in gastric carcinomas and regulates cell survival by targeting PDK1 and 14-3-3zeta. Cancer Res 2010;70:2339-49. 
33. Ladeiro Y, Couchy G, Balabaud C, et al. MicroRNA profiling in hepatocellular tumors is associated with clinical features and oncogene/tumor suppressor gene mutations. Hepatology 2008;47:1955-63.

34. Hui AB, Lenarduzzi M, Krushel $T$, et al. Comprehensive MicroRNA profiling for head and neck squamous cell carcinomas. Clin Cancer Res 2010;16:1129-39.

35. Zhou SL, Wang LD. Circulating microRNAs: novel biomarkers for esophageal cancer. World J Gastroenterol 2010;16:2348-54.
36. Chitnis MM, Yuen JS, Protheroe AS, et al. The type 1 insulin-like growth factor receptor pathway. Clin Cancer Res 2008;14:6364-70.

37. Dey BR, Furlanetto RW, Nissley SP. Cloning of human p55-gamma, a regulatory subunit of phosphatidylinositol 3-kinase, by a yeast two-hybrid library screen with the insulin-like growth factor-I receptor. Gene 1998;209:175-83.

38. Kota J, Chivukula RR, O'Donnell KA, et al. Therapeutic microRNA delivery suppresses tumorigenesis in a murine liver cancer model. Cell 2009;137:1005-17.

\section{Editor's quiz: GI snapshot}

\section{Constipation and recurrent abdominal distension in a 39 -year-old woman with irritable bowel syndrome}

\section{CLINICAL PRESENTATION}

A 39-year-old woman with a previous diagnosis of irritable bowel syndrome presented with progressively worsening episodes of marked abdominal distension and difficulties with defecation. She had a long history of constipation and had tried a variety of laxatives with an unsatisfactory response. Recent colonoscopy was unremarkable. Physical exam revealed a protuberant abdomen with tympany and high-pitched bowel sounds. Inspection of the anus showed no perianal lesions and absent perineal descent with straining at defecation. A digital rectal examination revealed high anal sphincter tone and absence of puborectalis relaxation with simulated defecation. There was no palpable stool in the rectum. Computed tomographic enterography with scout film showed a markedly dilated splenic flexure without obstructing mass or volvulus (Figure 1 and Figure 2). High-resolution anorectal manometry with balloon expulsion revealed high anal sphincter pressures, presence of the

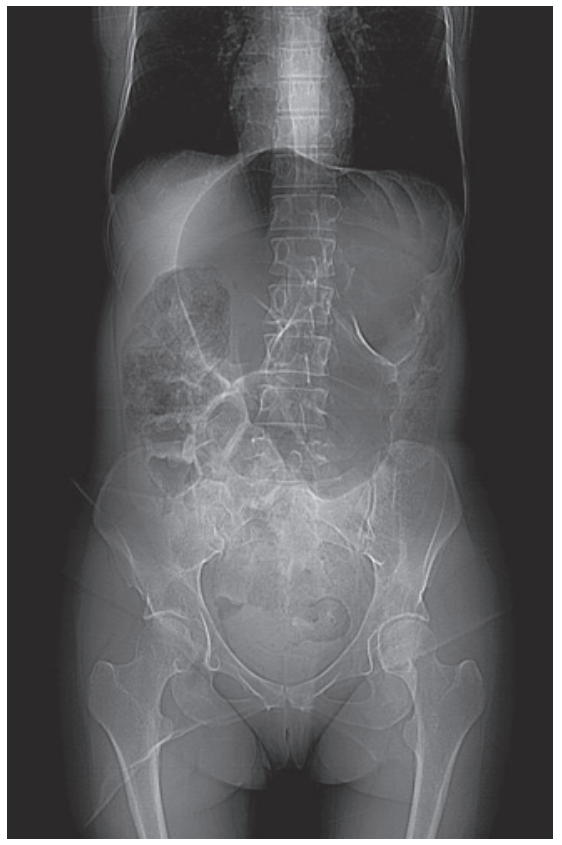

Figure 1 Scout film showing a markedly dilated splenic flexure.

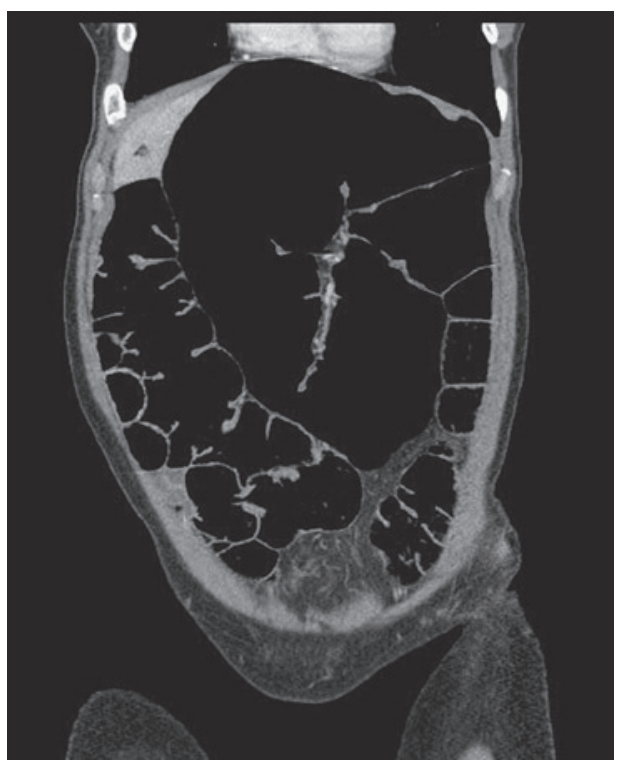

Figure 2 Computed tomographic enterography with a markedly dilated splenic flexure without obstructing mass or volvulus.

rectoanal inhibitory reflex and failure to expel the rectal balloon despite $564 \mathrm{~g}$ of traction (normal range $<200 \mathrm{~g}$ ).

\section{QUESTION}

What is the most likely diagnosis?

See page 107 for the answer

\section{Seth Sweetser, Archana S Rao, Lawrence A Szarka}

Division of Gastroenterology and Hepatology, Mayo Clinic College of Medicine, Rochester, Minnesota, USA

Correspondence to Dr Seth Sweetser, Mayo Clinic, 200 First St SW, Rochester, MN 55905, USA; sweetser.seth@mayo.edu

\section{Competing interests None.}

\section{Patient consent Obtained.}

Ethics approval This study was conducted with the approval of the Mayo Clinic College of Medicine. Institutional review board approval is not required for single case reports.

Contributors Seth Sweetser: design, acquisition of data and drafting of manuscript; Archana S. Rao: acquisition of data and drafting; Lawrence A. Szarka: critical revision of the manuscript for important intellectual content.

Provenance and peer review Not commissioned; internally peer reviewed.

Published Online First 2 September 2011

Gut 2012;61:42. doi:10.1136/gutjnl-2011-300925 


\section{GUT}

\section{MicroRNA-375 inhibits tumour growth and metastasis in oesophageal squamous cell carcinoma through repressing insulin-like growth factor 1 receptor}

Kar Lok Kong, Dora Lai Wan Kwong, Tim Hon-Man Chan, et al.

Gut 2012 61: 33-42 originally published online August 3, 2011

doi: $10.1136 /$ gutjnl-2011-300178

Updated information and services can be found at:

http://gut.bmj.com/content/61/1/33.full.html

\section{These include:}

References This article cites 35 articles, 10 of which can be accessed free at: http://gut.bmj.com/content/61/1/33.full.html\#ref-list-1

Email alerting Receive free email alerts when new articles cite this article. Sign up in service the box at the top right corner of the online article.

\footnotetext{
Topic Articles on similar topics can be found in the following collections

Collections

Oesophageal cancer (240 articles)
}

Notes

To request permissions go to:

http://group.bmj.com/group/rights-licensing/permissions

To order reprints go to:

http://journals.bmj.com/cgi/reprintform

To subscribe to BMJ go to:

http://group.bmj.com/subscribe/ 